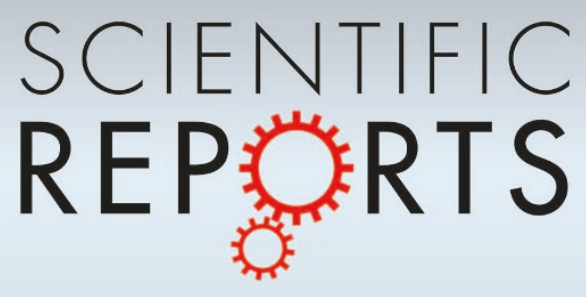

OPEN

SUBJECT AREAS:

BREAST CANCER

COMPUTATIONAL CHEMISTRY

VIRTUAL DRUG SCREENING

Received

18 March 2014

Accepted

2 June 2014

Published

20 June 2014

Correspondence and requests for materials should be addressed to L.A.E. (leif.eriksson@ chem.gu.se); N.F.L.

(noel.lowndes@ nuigalway.ie) or J.A.L.B. (james. brown@nuigalway.ie)

* These authors contributed equally to this work.

$\$$ ResearcherID: D-1226-2012.

$\uparrow$ Current address: Discipline of Surgery, School of Medicine, National University of Ireland, Galway, Ireland.

§ ORCID:

0000-0002-3155-

0334.

\section{Rational design and validation of a Tip60 histone acetyltransferase inhibitor}

\author{
Chunxia Gao ${ }^{1,2 *}$, Emer Bourke ${ }^{3 *}$, Martin Scobie ${ }^{4 *}$, Melina Arcos Famme ${ }^{4}$, Tobias Koolmeister ${ }^{4}$, \\ Thomas Helleday ${ }^{4}$, Leif A. Eriksson ${ }^{1,2}+$, Noel F. Lowndes ${ }^{5}$ \& James A. L. Brown ${ }^{5} \uparrow \S$
}

\begin{abstract}
'School of Chemistry, National University of Ireland Galway, Galway, Ireland, ${ }^{2}$ Department of Chemistry and Molecular Biology, University of Gothenburg, 405 30, Göteborg, Sweden, ${ }^{3}$ Discipline of Pathology, School of Medicine, Clinical Science Institute, Costello Road, National University of Ireland Galway, Galway, Ireland, ${ }^{4}$ Science for Life Laboratory, Division of Translational Medicine and Chemical Biology, Department of Medical Biochemistry and Biophysics, Karolinska Institute, Stockholm, Sweden, ${ }^{5}$ Genome Stability Laboratory, Centre for Chromosome Biology, School of Natural Sciences, National University of Ireland Galway, Galway, Ireland.
\end{abstract}

Histone acetylation is required for many aspects of gene regulation, genome maintenance and metabolism and dysfunctional acetylation is implicated in numerous diseases, including cancer. Acetylation is regulated by histone acetyltransferases (HATs) and histone deacetylases and currently, few general HAT inhibitors have been described. We identified the HAT Tip60 as an excellent candidate for targeted drug development, as Tip60 is a key mediator of the DNA damage response and transcriptional co-activator. Our modeling of Tip60 indicated that the active binding pocket possesses opposite charges at each end, with the positive charges attributed to two specific side chains. We used structure based drug design to develop a novel Tip60 inhibitor, TH1834, to fit this specific pocket. We demonstrate that TH1834 significantly inhibits Tip60 activity in vitro and treating cells with TH1834 results in apoptosis and increased unrepaired DNA damage (following ionizing radiation treatment) in breast cancer but not control cell lines. Furthermore, TH1834 did not affect the activity of related HAT MOF, as indicated by H4K16Ac, demonstrating specificity. The modeling and validation of the small molecule inhibitor TH1834 represents a first step towards developing additional specific, targeted inhibitors of Tip60 that may lead to further improvements in the treatment of breast cancer. 
mis-regulation or loss of ATF2, MDM2 or SIRT1 has been implicated in other forms of cancer ${ }^{14-19,29-36}$, suggesting a common mechanism of cancer progression.

Many cancer treatments use agents producing DNA damage, such as ionizing radiation (IR), to kill rapidly replicating cancer cells. Tip60 is critical for induction of the DNA damage response ${ }^{14-19}$. As such, we believe that Tip60 is a good candidate for the targeted design of a drug with the potential to become a chemotherapeutic. Only a small number of Tip60 inhibitors have been reported. The natural products anacardic acid and garcinol are the best known Tip60 inhibitors, and sensitize tumour cells to ionizing radiation ${ }^{37}$. Coupling a histone $\mathrm{H} 3$ peptide to $\mathrm{CoA}$ to form a bisubstrate compound can inhibit Tip60 activity as well, but this compound has low permeability $^{11,22}$. Other inhibitors include 6-alkylsalicylates ${ }^{9,23}$ and some small inhibitors reported by $\mathrm{Wu}$ and co-workers ${ }^{23,38}$. Recently, high throughput screening was used to identify a Tip60 inhibitor which demonstrated efficacy against prostate cancer cell lines by inducing apoptosis through caspase $3^{8,24}$. More recently, Pentamidine (PNT) has been reported to inhibit the activity of Tip60 by decreasing its histone H2A acetylation ${ }^{7}$. Although PNT has been used clinically against parasitic protozoan for over 70 years, it was only recently reported that DNA and protein synthesis in human tumours was decreased following PNT treatment, whereby PNT was proposed as an anti-tumour $\mathrm{drug}^{7}$.

Here we demonstrate the targeted design and synthesis of an inhibitor of Tip60, based on the scaffold of PNT and Acetyl-CoA. The compound, TH1834, was used to inhibit Tip60 activity in vitro and modulated a Tip60 dependent DNA damage response in vivo. To further understand the importance of Tip60 in breast cancer, characterisation of the inhibitor TH1834 was carried out primarily in breast cancer cells with low AR expression. To confirm our results, experiments were replicated in prostate cancer cells, where the role of Tip60 is more established ${ }^{23}$.

\section{Methods}

In silico modeling. Computational approaches. Homology modeling, induced fit docking, derivative structure building and interaction energy calculations were performed using the Molecular Operating Environment (MOE 2010.10) program (Chemical computing group Inc.). Molecular dynamics (MD) simulations were performed using the YASARA program ${ }^{39}$. For all protein-ligand complex systems a 20 ns MD simulation was performed to relax the system, followed by energy minimization and calculation of the interaction energy.

Homology modeling. The crystal structure of the acetyltransferase domain of human Tip60 complex with its natural ligand acetyl-CoA (PDB code: $2 \mathrm{OU} 2$ ) was taken from the protein data bank. However, since amino acid sequences 375-385 and 423-440 are missing from the crystal structure, a homology model of human Tip60 using the acetyltansferase domain sequence of human Tip60 (Supplemental Figure 1A) was built with the crystal structure of incomplete human Tip60 as template (PDB code: 2OU2). The homology model was constructed by taking the best of ten intermediate models, minimized to within an RMS gradient of 0.1 using the AMBER99 force field ${ }^{40}$.

Docking. PNT, which was found to suppress the activity of Tip60, was constructed using the molecular builder of MOE, hydrogen atoms were added, partial charges assigned and the structure energy minimized with the AMBER99 force field.

The ligand-binding site in the Tip60 homology model was identified using Alpha Site Finder. Once defined, PNT was docked into Tip60 with 500 poses retained, using the alpha triangle placement methodology with affinity $\Delta G$ as scoring function. In the docking studies, flexible ligand and receptor structures were generated using a Monte Carlo algorithm. The highest-ranking modeled ligand-protein interaction structure was selected, compared to the optimal binding of human Tip60 bound with AcetylCoA. Acetyl-CoA was also docked into the binding pocket of the homology model using the same method described above.

A set of PNT derivatives were then generated using the combinatorial fragment builder in MOE. PNT placed in the Tip60 binding pocket was used as the scaffold, and pocket atoms used to constrain the molecular construction. Three attachment sites of PNT were defined (Figure 1A), and functional groups from the default libraries connected to these. The best PNT derivative (TH1834) was selected after iterative design rounds, and then followed by $20 \mathrm{~ns}$ MD simulation and interaction energy calculations.

Molecular dynamics simulations. MD simulations were conducted with YASARA v10.7.20 $0^{39}$, using the AMBER03 ${ }^{41}$ force field. Partial atomic charges of ligands were computed using the AM1-BCC model $^{42}$ implemented in YASARA. MD simulations in explicit water were performed at constant temperature $(298 \mathrm{~K})$ after initial energy minimization procedures. Periodic boundary conditions were applied to all systems, and counter ions were added by randomly replacing water molecules by $\mathrm{Na}$ or $\mathrm{Cl}$ to provide a charge-neutral system and to give a total $\mathrm{NaCl}$ concentration of $0.9 \%$ corresponding to physiological solution. Long-range Coulomb interactions were included using particle-mesh Ewald (PME) summation ${ }^{43}$ and a cut-off of $7.86 \AA$. Simulations were carried out in their entirety, using a pre-defined macro (md_run) within the YASARA package. Multiple time steps were used in the simulation: $1.25 \mathrm{fs}$ for intramolecular and $2.5 \mathrm{fs}$ for intermolecular forces, and data were collected every 12.5 ps.

Interaction energy calculation. The interaction energies were calculated using the MM/GBVI implicit solvent method ${ }^{44}$ in the MOE programme. The interaction energy (IE) was defined as the energy difference between the enzyme-substrate complex (E-S) and individual enzyme (E) and substrate (S), according to Eqn 1:

$$
\mathrm{IE}=\mathrm{E}_{\mathrm{E}-\mathrm{S}}-\left(\mathrm{E}_{\mathrm{E}}+\mathrm{E}_{\mathrm{S}}\right)
$$

In order to eliminate the residual kinetic energy from the MD simulation, geometry optimizations were performed with the AMBER99 force field, and the MM/GBVI calculations performed on the geometries of the full enzyme-substrate complexes.

Ligand efficiency (LE) can be used to track the potency of fragment hits and to assess whether gains in potency are significant enough to justify increases in molecular size. LE is here defined as the interaction energy of a ligand to its receptor, per ligand atom, according to Eqn 2:

$$
\mathrm{LE}=-\mathrm{IE} / \mathrm{N}
$$

Where $\mathrm{N}$ is the number of heavy atoms in the ligand.

TH1834 synthesis. The final compound TH1834 was synthesized as described in Figures $2 \mathrm{~A}$ and $2 \mathrm{~B}$, and as detailed in Supplementary methods.

Cell culture conditions. The DT40 chicken B lymphocyte cell lines were cultured as previously described ${ }^{45}$. Briefly, DT40 cell lines were cultured at $39.5^{\circ} \mathrm{C}$ and $5.0 \% \mathrm{CO}_{2}$ in RPMI 1640 and 10\% foetal bovine serum (FBS) [Lonza (Berkshire, UK)], 1\% chicken serum, $100 \mathrm{U} / \mathrm{ml}$ penicillin and $100 \mu \mathrm{g} / \mathrm{ml}$ streptomycin [Sigma (Dublin, Ireland)]. All human cell lines (MCF7, MCF10A, PWR-1E, DU145 and PC-3) were cultured at $37^{\circ} \mathrm{C}$ and $5.0 \% \mathrm{CO}_{2}$ in the appropriate media as defined by the ATCC. All cell lines were originally purchased from ATCC, with the Breast cancer cell lines a kind gift from Dr E. Szegezdi and Prof A. Samali (National University of Ireland Galway, Ireland) and the prostate cancer cell lines a kind gift from Prof C. Morrison (National University of Ireland Galway, Ireland).

Kat5 (Tip60) gene identification in Gallus gallus. The human Kat5 gene sequence (KC355247) was used to search the Gallus gallus genome (NCBI: BLAST), producing a number of hits on Gallus gallus EST clones. The sequence identified in these clones were found to have significant homology $(>80 \%)$ to human Kat5, and these new sequences were then used in subsequent BLAST searches of the Gallus gallus genome, retrieving further sequences. Together these sequences were assembled into one contiguous 1,263 nt long putative Kat5 sequence using DNA strider ${ }^{46}$.

Kat5 amplification and cloning. mRNA was extracted from DT40 cells with Tri Reagent (Sigma) as per manufacturer's instructions. cDNA was synthesized from the extracted mRNA using SuperScript (Life Technologies) as per manufacturer's instructions. Kat5 primers were used in a cDNA PCR reaction with Pfu (Stratgene, USA), as per manufacturer's instructions, which amplified DNA of the expected size (Figure S2A). Primers were designed against the putative Kat5 sequence; forward 5'-ATGGCGGAGGCGGCC-3' with an EcoR1 site and reverse 5' - CACCACTTGCCCCTCTTG-3' with Kpn1 site. The amplified Kat5 sequence (1,391 nt) was verified by sequencing and varied slightly from the predicted sequence. The verified sequence was deposited in the NCBI database (KC355247).

GFP-tagged Tip60 vectors cloning and stable transfection. Tip60 was cloned into a pEGFP-N1 vector (Clonetech) containing a Neomycin resistance cassette and the final construct verified by sequencing. The Tip60-GFP construct was randomly integrated into the genome of WT DT40 cells as previously described ${ }^{47}$. Expression of GFP-tagged Tip60 was verified by Western blot (Figure 3A) and by immunofluorescence detection of GFP (Figure 3B, S4).

Antibodies. Western blotting was performed using the antibody recognizing antiacetyl lysine (Millipore; AB3879), anti-GFP (Roche; 11814460 001), Anti-Tip60 (K17, Santa Cruz), Anti- $\beta$ Tubulin (Ab6046, Abcam), $\beta$-actin (Sigma), Cleaved caspase 3 (Asp175) (91164; Cell Signalling), H4K8 (Millipore; 07-328), H4K16 (Millipore; 07-329), H3 (Abcam; AB1791). Immunofluorescent detection of $\gamma \mathrm{H} 2 \mathrm{AX}$ was performed using Antibody JBW301 (Millipore) and 53BP1 (Bethyl Laboratories; A300-272A) with a Texas Red and FITC-conjugated secondary antibodies respectively (Jackson Immunoresearch).

Immunoblotting. Whole cell lysates were prepared using RIPA buffer containing a protease inhibitor cocktail (Roche). Whole cell lysates were re-suspended in $(1: 4 \mathrm{v} / \mathrm{v})$ reducing sample buffer, boiled and separated by SDS-PAGE. Proteins were transferred to nitrocellulose membranes (Life Technologies) by standard procedures. 


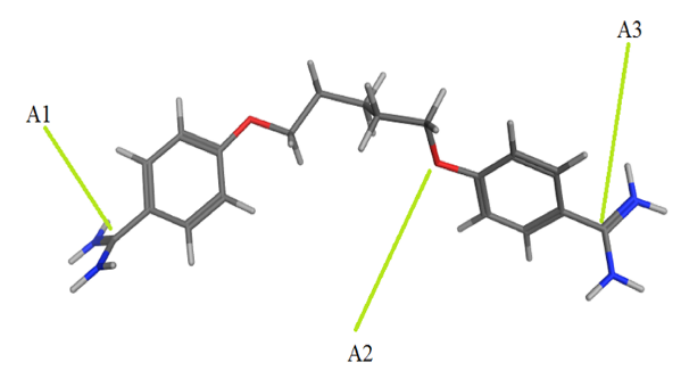

C

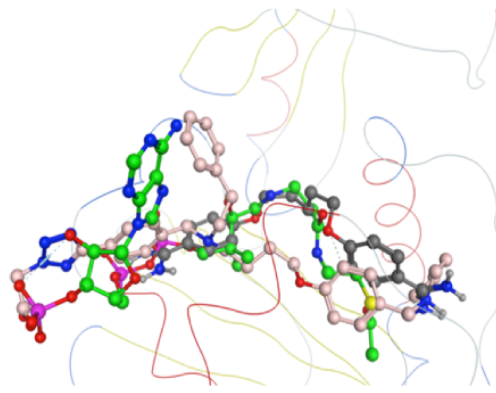

E

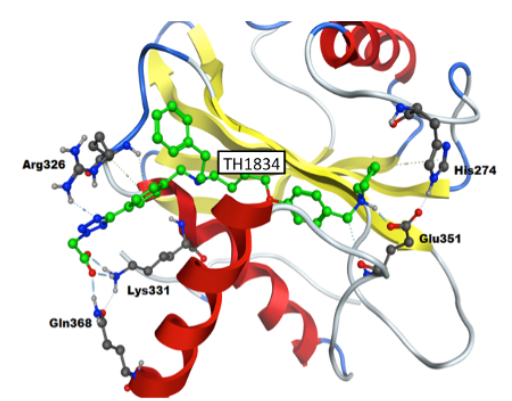

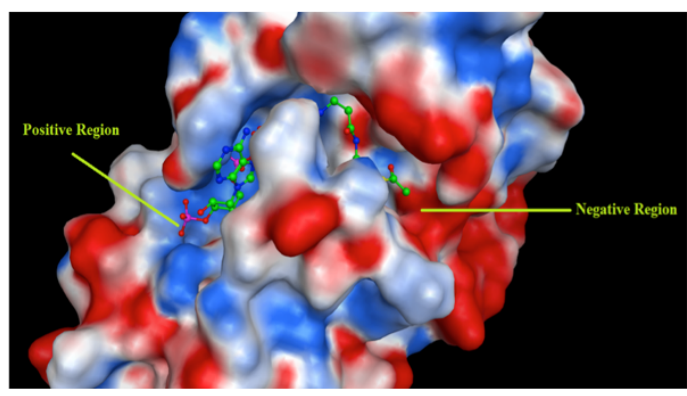

$\mathrm{D}$

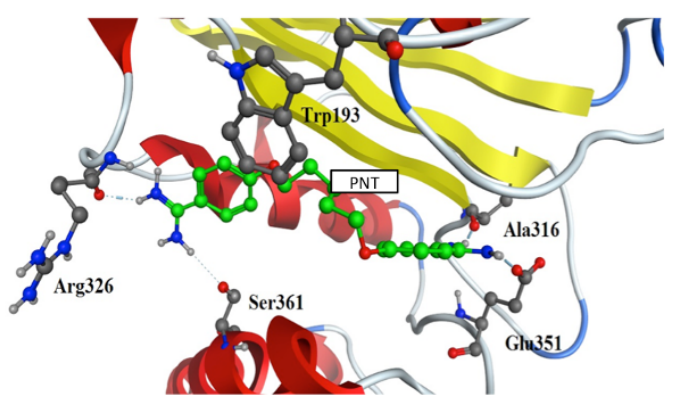

$\mathrm{F}$

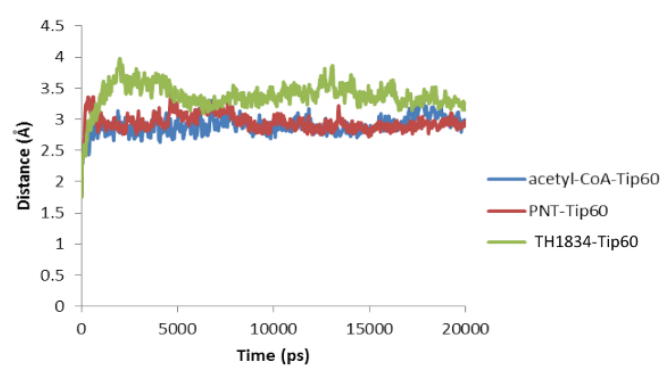

Figure $1 \mid$ In silico modeling of TH1834 bound to Tip60. (A). Attachment points of PNT in the combinatorial builder approach. (B). Superposition of homology model and crystal structure of Tip60 acetyltransferase domain. (C). Acetyl-CoA, PNT and TH1834 bound into the Tip60 binding pocket. (D). PNT in the binding pocket of Tip60. (E). Detailed interaction of TH1834 in the Tip60 binding pocket. (F). RMSDs of the MD simulations of the complex systems.

Membranes incubated overnight at $4^{\circ} \mathrm{C}$ with the indicated primary antibodies in $5 \%$ skim milk powder in TBS/0.1\% Tween-20 (TBS/T). Membranes were incubated for $1 \mathrm{hr}$ at room temperature with HRP conjugated secondary antibodies (Jackson ImmunoResearch Laboratories Inc.) in 5\% skim milk powder/TBS/T followed by washing in TBS/T. Bound antibodies were visualized using SuperSignal West Pico Chemiluminescent substrate (Thermo Pierce Scientific). Band intensities were quantified using ImageJ software. Integrated density readings, or pixel intensities, were measured using the manual region of interest (ROI) tool. A region of interest (ROI) was drawn on the caspase 3, H4K8ac and H4K16ac signals and the ROI measured as grey values. For quantification, the loading ROI (L-ROI) were measured in the corresponding $\mathrm{H} 3$ or $\beta$-actin lanes and the caspase 3, H4K8ac and H4K16ac ROI values normalized to these L-ROI values. Quantification of caspase 3, H4K8ac and $\mathrm{H} 4 \mathrm{~K} 16 \mathrm{ac}$ was performed by setting each normalized ROI in the untreated lane to $=1$ and subsequent treatment ROI values calculated compared to this.

Immunofluorescence microscopy. Cells were prepared and stained as previously described ${ }^{2,3,45}$. In brief, cells were fixed in $4 \%$ paraformaldehyde followed by permeabilisation in $0.1 \%$ Triton-X-100 and then blocked with 5\% FCS. Cells were incubated with the indicated primary antibodies overnight at $4{ }^{\circ} \mathrm{C}$, washed and incubated with alexa fluor conjugated secondary antibodies. Images were acquired with an Olympus BX51 microscope and Openlab software (Improvision). $0.4 \mu \mathrm{m}$ $Z$-stacks were collected and brightest point projections created from stacked sections. $\gamma-\mathrm{H} 2 \mathrm{AX}$ foci counted using automated quantification by Cell Profiler software ${ }^{4-6,48}$ with automated counts manually verified.

In vitro histone acetylation assay. Cells were lysed $(50 \mathrm{mM}$ Tris $\mathrm{HCl} \mathrm{pH} 8.0$, $150 \mathrm{mM} \mathrm{NaCl}, 5 \mathrm{mM}$ EDTA, $0.5 \% \mathrm{NP}-40$ with protease inhibitors, $1 \mathrm{mM}$ each DTT, PMSF and NaF) for 1 hour at $4{ }^{\circ} \mathrm{C}$. Cellular debris was pelleted and the GFP-tagged
Tip60 immunoprecipitated using GFP-Trap beads (ChromoTek GmbH). Pellets were washed with HAT assay buffer (50 mM Tris HCL pH 8.0, 10\% Glycerol, $100 \mu \mathrm{M}$ Acetyl-CoA and protease inhibitors, $1 \mathrm{mM}$ each DTT and NaF). The HAT assay was performed in HAT assay buffer with the addition of $1 \mu \mathrm{g}$ of recombinant $\mathrm{H} 2 \mathrm{~A}$, and TH1 834 where indicated, for $30 \mathrm{~min}$ at $30^{\circ} \mathrm{C}$. Reactions were stopped with $2 \times$ SDS loading buffer.

Viability, cytoxicity and caspase activation assay. Effect of TH1834 on viability, cytotoxicity and caspase 3 activation was assayed using the ApoTox-Glo ${ }^{\mathrm{TM}}$ Triplex Assay (Promega) as per the manufacturer's instructions, under the indicated conditions.

Statistical analyses. To avoid any bias, counts were recorded from multiple fields from the indicated number of independent cell culture preparations. Data analyses were performed using Prism 5 software (GraphPad Software, Inc., La Jolla, CA, USA). Statistical significance was calculated with a One way ANOVA test with a posthoc Dunnes test to asses statistical significance between groups, unless otherwise indicated. A P-value $<0.05\left({ }^{*}\right)$ was deemed significant, $\mathrm{P}<0.01(* *)$ very significant and a $\mathrm{P}<0.001(* * *)$ highly significant, as stated in the figure legends. Values of counts Foci counts represent at least 3 separate experiments with $>30$ measurements/counts made per independent experiment ( $\mathrm{n}$ and minimum number measurements per experiment indicated in figure legends). All graphs display mean $(+/-$ SEM).

\section{Results}

Gallus gallus Tip60 identification and verification. The Gallus gallus Tip60 (Kat5 gene) was putatively identified by a BLAST 


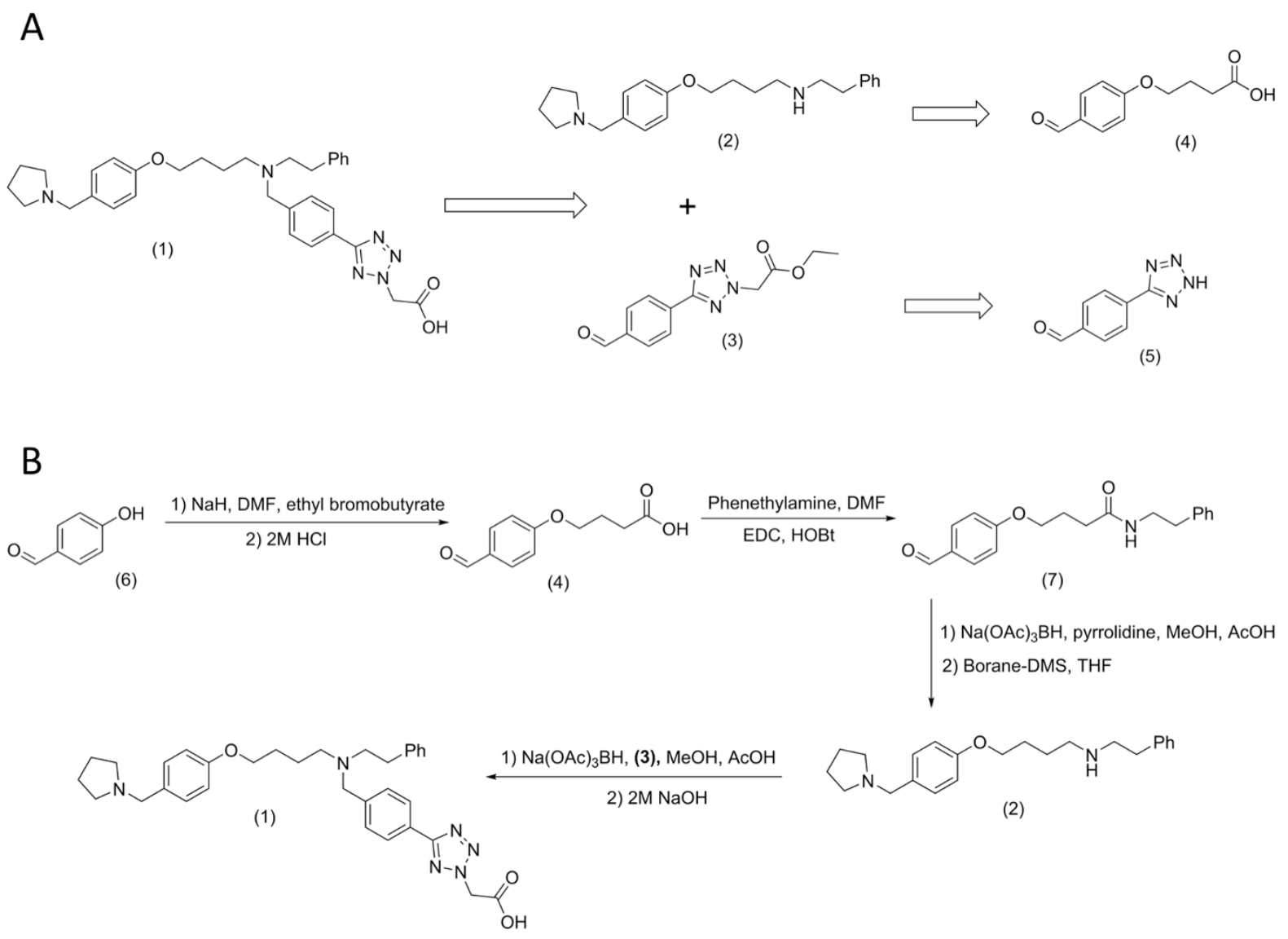

Figure $2 \mid$ Synthesis of TH1834. (A). Retrosynthetic strategy for synthesis of the TIP60 inhibitor TH1834. (B). Synthesis of TH1834.

(NCBI) search using the human Kat5 sequence against the Gallus gallus NCBI mRNA database. This identified a number of EST clones with high homology, which were then used to identify further EST sequences. The EST sequences were aligned and a putative full length Gallus gallus Kat5 mRNA sequence identified, from which a putative full length Kat5 sequence was assembled (Figure S1). Using this sequence, primers were designed and the full length Kat5 sequence amplified (Figure S2A). The amplified DNA was sequenced, producing a full-length Kat5 sequence (varying slightly from the predicted sequence) which was deposited at NCBI (KC355247). There was high sequence homology $(81 \%)$ and identity $(83 \%)$ between isolated DT40 Kat5 and the human Kat5 isoform 1 sequence (Figure S2B: nucleotide and S2C: protein), suggesting that the isolated Gallus gallus Kat5 is functional (Figure S2D) ${ }^{7-11,49}$. However, the overall value underrepresents the homology, due to Gallus gallus Kat5 missing regions present in the human sequence. The catalytic HAT domain of chicken Tip60 contains 97\% identity with human Tip60 ( $3^{\prime}$ region of the sequence), with only 9 amino acid differences (Figure S3).

Kat5 was cloned into a GFP-tagged expression vector for further analysis. GFP-tagged Tip60 was over-expressed and was functional, as GFP-Tip60 re-localised to sites of ionizing radiation-induced DNA damage marked by co-localisation with the marker of DNA double strand breaks, gamma $\mathrm{H} 2 \mathrm{AX}$, as previously described ${ }^{3,14}$ (Figure S4).

Modelling of ligands bound to Tip60. Initially, the main interaction sites between Tip60 and the inhibitor PNT were defined, and used as references for further modeling (Figure 1A). The complete homology model of Tip60 we obtained superposes well with the incomplete template crystal structure of Tip60, with the overall Ca RMSD being $0.535 \AA$. The complete homology model of Tip 60 has an additional two residues (Arg434 and Leu437) that contribute to the interaction with acetyl-CoA, compared to the incomplete crystal structure (Figure 1B). The electrostatic surface potential of the binding pocket shows that one end of the binding pocket is mainly positive whereas the other end is negative (Figure 1B). The positive region arises from the side chains of Arg326, Lys331 and Arg434, and the negative region is from the side chain of Glu351, as well as the backbone carbonyls of residues Lys352 and Pro353. Arg326, Lys331 and Arg434 interact with the phosphate group of acetyl-CoA, Glu351 is located towards the end of the long tail of acetyl-CoA, and is not hydrogen bonding to acetyl-CoA. It has, however, been proposed to function as a general base for the catalysis of histone lysine ${ }^{12-15,50}$.

The previously described compound PNT has been found to inhibit the ionizing radiation induced activity of Tip $60^{7,16}$. From the docking study, the PNT docked pose superposes well with the original acetyl-CoA ligand (Figure 1C), with one of its protonated amidine lying in a deep pocket in the negative region and forms a network of hydrogen bonds with the side chain Glu351 and the backbone carbonyl of Ala316. The other protonated amidine forms a network of hydrogen bonds with side chain Ser361 and backbone carbonyl of Arg326 (Figure 1D). In order to increase the binding efficiency of PNT, the chemical structure of the new ligand (TH1834) was designed with a resulting negative carboxylic group predicted to interact with the positive region of the binding pocket, and a protonated pyrrolidine group to interact with the negative region. A long bulky ethylbenzene side chain was added to the main chain of the compound to mimic the location of the adenine group in acetyl-CoA (Figure 1B). We see that the carboxylic group of TH1834 indeed interacts with Lys331 and Gln368, and the pyrrolidine group interacts with Glu351 and His 274 (Figure 1E). More detailed interactions of acetyl-CoA, PNT and TH1834 with the various pocket residues are shown in supplementary Figure S5(A-C). 
A

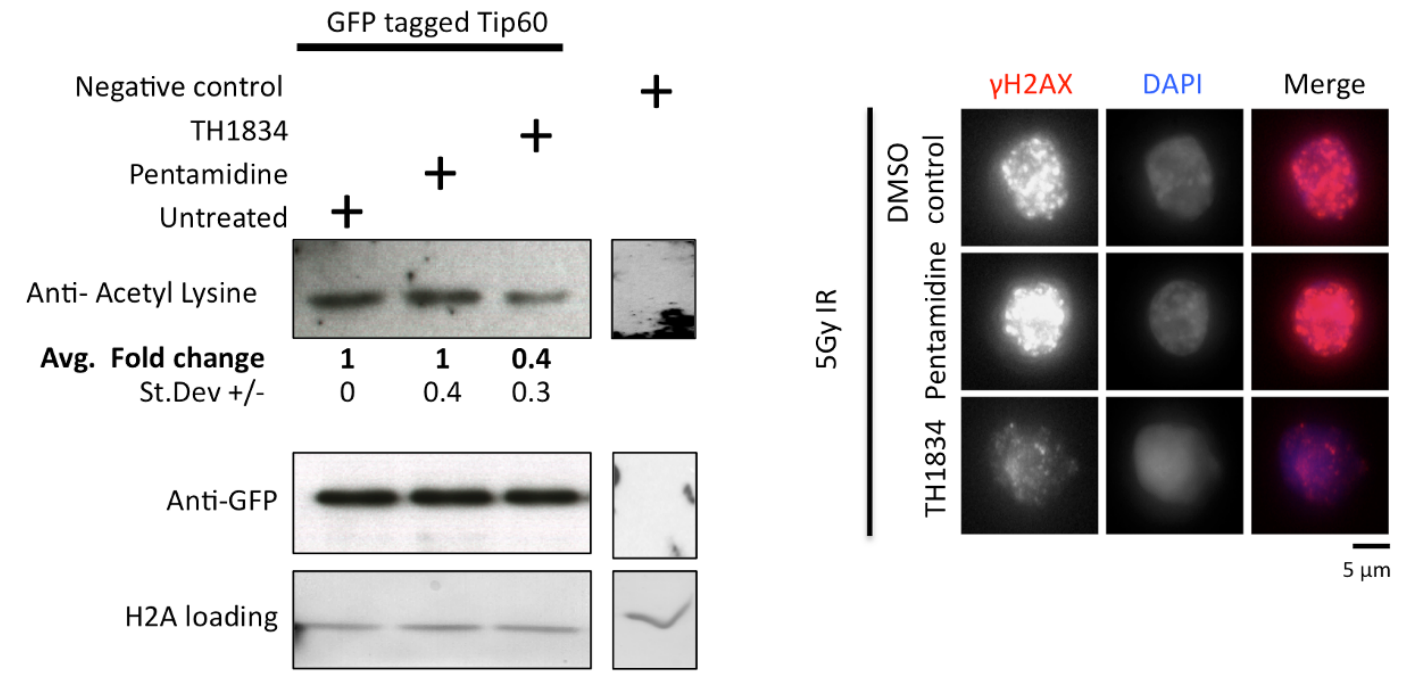

C

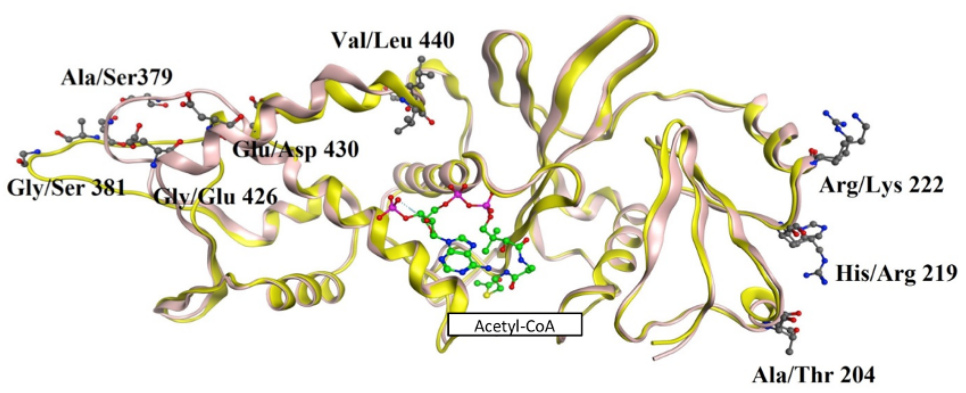

Figure 3 TH1834 inhibits Tip60 activity. (A). TH1834 inhibits Tip60 activity in vitro. Untreated DT40 cells were lysed and GFP-tagged Tip60 immunoprecipitated and used for an in vitro histone acetylation assay. TH1834 (500 $\mu \mathrm{M})$ or PNT $(500 \mu \mathrm{M})$ were added for the final assay. Negative control: IP from non-transfected control cells. Quantification (average) of fold change in activity (normalized to untreated) and standard deviation indicated below the acetyl lysine blot. Cropped gels/blots are shown. (B). TH1834 but not PNT inhibits $\gamma \mathrm{H} 2 \mathrm{AX}$ formation in vivo. DT40 cells were pretreated with $500 \mu \mathrm{M}$ of TH1834 or PNT for $1 \mathrm{hr}$ prior to 10 Gy IR treatment. Cells were fixed $1 \mathrm{hr}$ post IR treatment. Scale bar $5 \mu \mathrm{m}$. (C). 3D alignment and homology modeling of acetyl-CoA bound chicken Tip60 (yellow ribbon) and human Tip60 (pink ribbon) HAT domains. Non-homologous amino acids (labeled), located outside the acetyl-CoA substrate-binding site.

Molecular dynamics simulations were performed on the ligandprotein complex systems acetyl-CoA-Tip60, PNT-Tip60, TH1834Tip60 respectively. The stability of each simulation system was evaluated based on its root mean square deviation (RMSD). The RMSD values for the three complex systems versus simulation time are illustrated in Figure 1F. The RMSD for TH1834-Tip60 is initially a little higher than that of the other two systems due to rearrangements caused by the bulky side group added, but all systems equilibrate within $5 \mathrm{~ns}$ and are stable thereafter. IE and LE were calculated for the complex systems. Although acetyl-CoA has a larger IE $(-46.9 \mathrm{kcal} / \mathrm{mol})$, it displays a smaller LE $(-0.92)$ than TH1834. The IE of TH1834 $(-40.3 \mathrm{kcal} / \mathrm{mol})$ is much larger than that of PNT, and the LE of -0.96 (Table 1) demonstrates it to be comparable to acetyl-CoA in binding to Tip60, though it is relatively smaller than LE of PNT (-1.30). In agreement with this, TH1834 was also shown experimentally herein to elicit higher inhibitory activity than PNT.

Synthesis of TH1834. We envisaged a convergent synthesis (Figure 2A) of the target compound TH1834 (1) using reductive alkylation and as the key synthetic step to link the aryltetrazole aldehyde containing fragment (3) with a secondary amine (2). The synthesis of TH1834 is detailed in the supplementary methods.
Briefly, synthesis of the required aldehyde (3) was readily achieved by alkylation of commercially available 4-(2H-1,2,3,4-tetrazol-5yl)benzaldehyde (5) with ethyl bromoacetate using sodium hydride as base. These conditions afforded (3) in reasonable yield (54\%) and were not further optimised. The amine containing fragment (2) was prepared in five synthetic steps as outlined in Figure 2B. Alkylation of

\begin{tabular}{|lccc|}
\hline $\begin{array}{l}\text { Table } 1 \text { |Structures and interaction } \\
\text { compounds } \\
\text { Name }\end{array}$ Structure & $\mathrm{IE}(\mathrm{kcal} / \mathrm{mol})$ & $\mathrm{LE}$ \\
\hline $\mathrm{PNT}$ & -32.52 & -1.30 \\
$\mathrm{TH} 1834$ & -40.28 & -0.96 \\
\hline
\end{tabular}


4-hydroxybenzaldehyde (6) with ethyl bromobutyrate followed by in situ hydrolysis of the ester afforded the desired acid (4) in good yield (65\%, over two steps). The resulting acid (4) was then coupled with phenethylamine using standard $\mathrm{EDC} / \mathrm{HOBt}$ conditions to give the amide (7) in high yield (72\%). At this stage the benzylic pyrrolidine motif was introduced by reductive amination of (7) with pyrrolidine using sodium triacetoxyborohydride in dichloroethane ( $84 \%$ yield). Sequential reduction of the amide linker using boranedimethylsulphide in THF gave access to the required secondary amine (2), albeit in low yield (28\%).

Finally, reductive alkylation of the tetrazole-aldehyde (3) with amine (2), followed by base-catalysed hydrolysis of the ester group, gave the target compound ( 1 ; TH1834) in high yield and purity $(82 \%$ yield, over two steps). The final compound had spectroscopic and analytical data in full agreement with the assigned structure (Figure S6A-B).

TH1834 activity. To determine if TH1834 directly inhibited Tip60 activity, GFP-tagged Tip60 was used to facilitate expression and purification. GFP-Tip60 was expressed in DT40 cells, immunoprecipitated and used in an in vitro histone acetyl-transferase (HAT) assay (Figure 3A). In our HAT assay, we found no inhibition of Tip60 activity by the non-specific HAT inhibitor PNT. Following TH1834 treatment we observed a significant $(60 \%)$ [P-value $<0.05(*)$ ] reduction in Tip60 activity, as demonstrated by the quantification (Figure 3A, S7).

Tip60 acetylation is required for activation of the ATM kinase and phosphorylation of $\mathrm{H} 2 \mathrm{AX}(\gamma \mathrm{H} 2 \mathrm{AX})$ following DNA double strand breaks ${ }^{1,14,15,17-19}$. Therefore, the appearance of $\gamma \mathrm{H} 2 \mathrm{AX}$ foci can be used as a reporter of Tip60 activity in vivo ${ }^{2,3,6-11,14,15,20}$. DT40 cells were pre-treated with $500 \mu \mathrm{M}$ of either PNT or TH1834 for 1 hour prior to exposure to $5 \mathrm{~Gy}$ of ionizing radiation (Figure $3 \mathrm{~B}$ ). Significant inhibition of the appearance of $\gamma \mathrm{H} 2 \mathrm{AX}$ foci was observed in cells pre-treated with TH1834 but not PNT. 3D modeling of the chicken and human HAT domains indicated the identified 9 amino acid differences lie outside the substrate-binding site (Figure 3C) and are unlikely to result in any significant effects. Due to the high overall homology observed between the chicken and human Tip60 catalytic domain (97\%, Figure 3C, S2-3), further work was carried out investigating the effect of TH1834 on human Tip60.

Tip60 expression has been implicated in both prostate and breast cancer ${ }^{23,24}$ and most recently its role was directly investigated in AR low prostate cancer cells ${ }^{8,22}$. We therefore chose to focus on the effect of TH1834 in breast cancer cells with low/null AR expression $(\mathrm{MCF} 7)^{27,28}$, with further confirmation in the AR negative prostate cancer cell lines DU-145 and PC-3. In both the MCF10A control non-tumorigenic myoepithelial cell line and the MCF7 breast cancer cell line, 2 Gy IR treatment induced an increase in $\gamma \mathrm{H} 2 \mathrm{AX}$ foci formation (Figure 4A). The combination of TH1834 pre-treatment and IR treatment resulted in a reduction in $\gamma \mathrm{H} 2 \mathrm{AX}$ (Figure 4A, left side) and 53BP1 foci (Figure 4B, left side) in control MCF10A cells. However in the MCF7 breast cancer cells, an increase in both $\gamma \mathrm{H} 2 \mathrm{AX}$ (Figure $4 \mathrm{~A}$, right side) and 53BP1 foci (Figure $4 \mathrm{~B}$, right side) was observed.

Quantification of $\gamma \mathrm{H} 2 \mathrm{AX}$ foci in response to TH1834 alone revealed no significant difference in MCF10A cells (Figure 4C, left side). However, the cancer cell line MCF7 displayed a significant increase in $\gamma \mathrm{H} 2 \mathrm{AX}$ foci in response to treatment with $50 \mu \mathrm{M}[\mathrm{P}<$ $0.001(* * *)]$ and $500 \mu \mathrm{M}[\mathrm{P}<0.01(* *)] \mathrm{TH} 1834$ (Figure $4 \mathrm{D}$, left side).

Following 2 Gy IR treatment, both MCF10A and MCF7 demonstrated a significant $[\mathrm{P}<0.01(* *)]$ increase in $\gamma \mathrm{H} 2 \mathrm{AX}$ foci (Figure 4C-D). Furthermore, MCF10A IR treated cells displayed a highly significant $[\mathrm{P}<0.001(* * *)]$ increase in $\gamma \mathrm{H} 2 \mathrm{AX}$ IRIF, compared to treatment with 50 or $500 \mu \mathrm{M}$ TH1834 (Figure 4C). Pretreatment of the control cell line MCF10A with $500 \mu \mathrm{M}$ TH1834, prior to exposure to 2 Gy IR resulted in a significant reduction $[\mathrm{P}<$ $0.01(* * *)]$ in $\gamma \mathrm{H} 2 \mathrm{AX}$ foci (Figure $4 \mathrm{C}$, right side). Conversely, in the breast cancer cell line MCF7 the combination of TH1834 and IR resulted in a highly significant $[\mathrm{P}<0.001(* * *)]$ increase in $\gamma \mathrm{H} 2 \mathrm{AX}$ foci (Figure 4D, right side). In both instances the effect of TH1834 was dose dependent. Additionally, investigating the effect of the combination of TH1834 and IR in prostate cancer cells revealed an increase in $\gamma \mathrm{H} 2 \mathrm{AX}$ foci in the cancer cell lines PC-3 and DU-145, in agreement with previous studies using prostate cancer cells $s^{8,22}$ (Figure S8, upper panels). Furthermore, supporting our previous findings in the control breast cell line, TH1834 pre-treatment combined with IR resulted in a decrease in $\gamma \mathrm{H} 2 \mathrm{AX}$ foci in the control prostate cell line PWR-1E (Figure S8, lower panels).

TH1834 mechanism of action. To investigate the mechanism of action of TH1834 activation of the apoptotic pathway was monitored, through the cleavage and activation of caspase 3 in the luminal breast cancer cells MCF7 and the non-tumorigenic myoepithelial (basal) MCF10A breast cells (Figure 5A). Sensitivity to TH1834 $(500 \mu \mathrm{M})$ was observed due to the appearance of cleaved caspase 3 in the breast cancer cells MCF7 ( $\sim 29$ fold increase), with the control cell line MCF10A unchanged (Figure 5A). Additionally, following TH1834 treatment marked caspase 3 activation was observed in MCF7 but not MCF10A cells in an independent assay (Figure S9A-B). Investigating specificity, the pattern of $\mathrm{H} 4 \mathrm{~K} 8 \mathrm{ac}$ was slightly increased (compared to untreated) by pre-treatment with TH1834, both in the absence and presence of IR (Figure 5B). Basal hMOF dependent H4K16ac was unaffected by TH1834 and IRdependent induction of H4K16Ac still occurred in the presence of TH1834 (Figure 5C).

Investigating the dose response of $\mathrm{TH} 1834$, treatment significantly reduced the viability of MCF7 cells at the highest concentrations (Figure 5D) with a corresponding highly significant increase in cytotoxicity at all concentrations used (Figure 5E). However, TH1834 did not significantly affect the viability of MCF10A cells at the highest concentration $(500 \mu \mathrm{M})$, which was used in Figure 4A (Figure 5D). In addition, TH1834 only increased cytotoxicity in the control MCF10A cells at $500 \mu \mathrm{M}$. However, $500 \mu \mathrm{M}$ TH1834 induced a highly significant increase in cytotoxicity in MCF7 cells, compared to MCF10A (Figure 5E). Staurosporine, a known inducer of apoptosis, was included as a positive control inducing significant cytotoxicity (Figure 5E and S9). Furthermore, $500 \mu \mathrm{M}$ TH1834 induced significantly more cytotoxicity than staurosporine. The combination of IR and TH1834 treatment of the prostate cancer (DU-145) cell line also induced appearance of a sub-G1 peak (Figures S10) indicating cell death, supporting the results from Figure 5.

\section{Discussion}

Tip60 plays important roles in chromatin remodeling, genomic stability and in initiating the DNA double strand break response ${ }^{12-15,23}$. Inhibitors of Tip60 therefore have great potential in cancer treatment, however development of targeted small molecule Tip60 inhibitors is still in the very early stages. Our study demonstrates a proof-of-principle, that targeted modifications of PNT (a previously reported inhibitor ${ }^{7,23}$ ) using in-silico modeling is possible. As Tip60 is associated with androgen receptor activity, breast cancer cells with reportedly low expression of the androgen receptor were chosen for this study ${ }^{27,28}$. Interestingly, we found that breast cancer cells MCF7 were more sensitive to TH1834 (increased low dose IR induced DNA damage, caspase 3 activity, cytotoxicity and/or cleavage;), compared to the non-tumorigenic line MCF10A (Figures 3-5 and S8). This was supported by the TH1834-induced IR sensitivity observed in the cancer prostate cancer cell line DU-145 (Figure S8 and 10).

We designed a targeted ligand (TH1834), which demonstrated inhibitory activity against Tip60 and Tip60-dependent signalling 


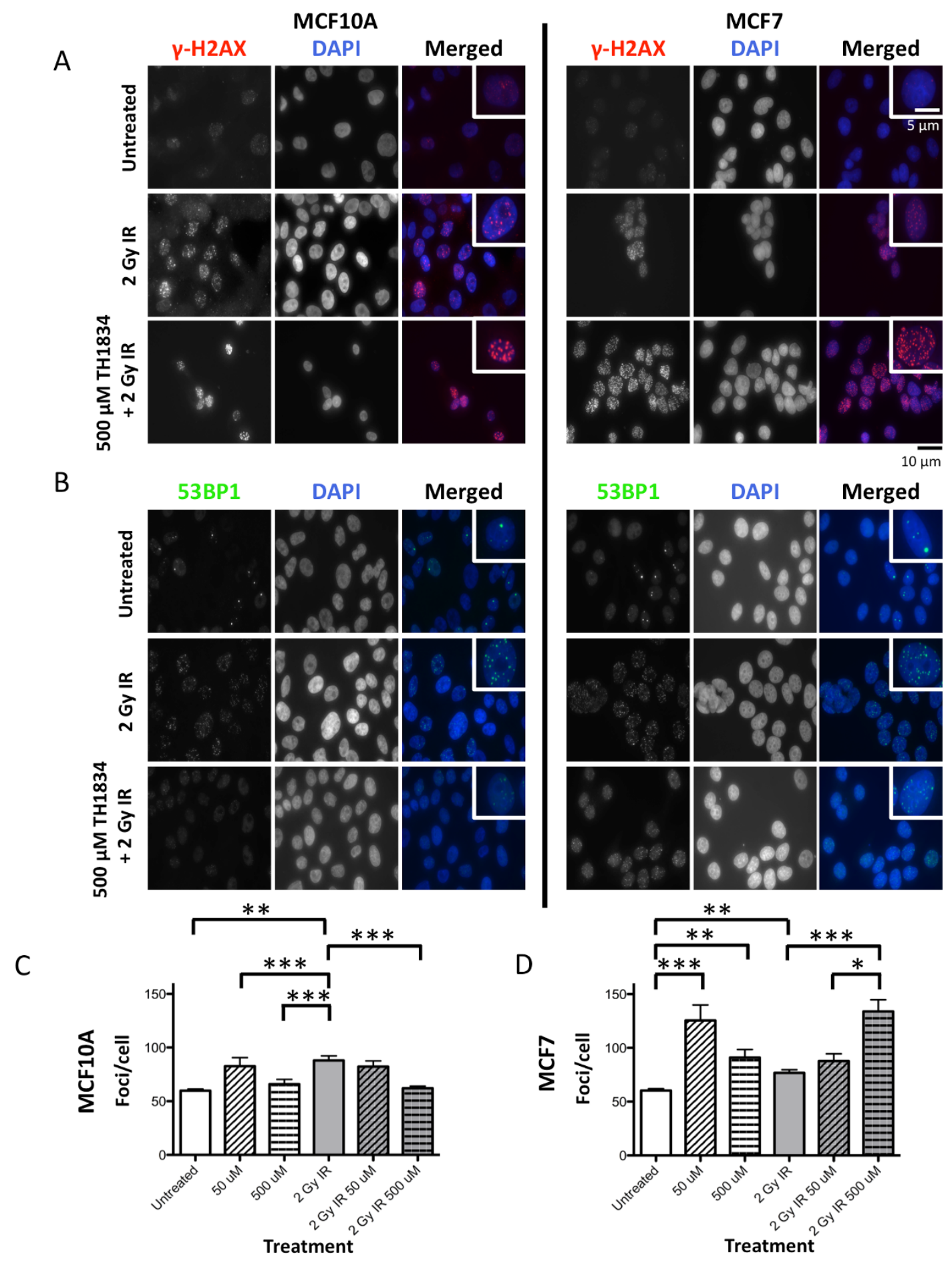

Figure $4 \mid$ TH1834 inhibits Tip60 dependent signalling. (A). TH1834 induces $\gamma \mathrm{H} 2 \mathrm{AX}$ IRIF. Indicated Breast cancer cell lines pre-treated with $500 \mu \mathrm{M}$ of TH1834 for $1 \mathrm{hr}$ prior to 2 Gy IR treatment and stained for $\gamma \mathrm{H} 2 \mathrm{AX}$ (Red) and DNA (Blue). (B). TH1834 reduces 53BP1 IRIF. Indicated Breast cancer cell lines pre-treated with $500 \mu \mathrm{M}$ of TH1834 for $1 \mathrm{hr}$ prior to 2 Gy IR treatment and stained for 53BP1 (Green) and DNA (Blue). (A-B). Scale bar $10 \mu \mathrm{m}$. Merged image insert: enlargement of individual nuclei. Insert scale bar $5 \mu \mathrm{m}$. (C-D). Quantification of TH1834 affects on $\gamma \mathrm{H} 2 \mathrm{AX}$ formation.

(C). Pre-treatment with TH1834 reduces $\gamma \mathrm{H} 2 \mathrm{AX}$ foci following IR treatment in control MCF10A cells. (D). Pre-treatment with TH1834 increases $\gamma \mathrm{H} 2 \mathrm{AX}$ foci following IR in MCF7 cancer cells. $\gamma \mathrm{H} 2 \mathrm{AX}$ foci quantified by automated counting using CellProfiler. Statistical analysis: One way Anova KruskalWallis analysis with Dunns post test. Graphs represents $\mathrm{N}=3$ ( $>30$ cells evaluated per independent experiment). A P-value $<0.05(*)$ was deemed significant, $\mathrm{P}<0.01(* *)$ very significant, and a $\mathrm{P}<0.001(* * *)$, highly significant.

both in vivo and in vitro when combined with IR (Figures 3-5, S7). Furthermore, highlighting the importance of Tip60 in cancer, its inhibition prior to IR treatment resulted in significantly increased numbers of $\gamma \mathrm{H} 2 \mathrm{AX}$ IRIF in the cancer cell line MCF7, but not in the control cell line MCF10A (Figure 4A). The same TH1834 dependent trend was observed in the prostate cell lines, with the cancer lines DU-145 and PC-3 displaying increased $\gamma \mathrm{H} 2 \mathrm{AX}$ IRIF and the control line, PWR-1E demonstrating a reduction in $\gamma \mathrm{H} 2 \mathrm{AX}$ foci (Figure S8). It has been proposed that haploinsufficiency of Tip60 accelerates cellular progression towards a tumorigenic phenotype ${ }^{24}$. This is 
A
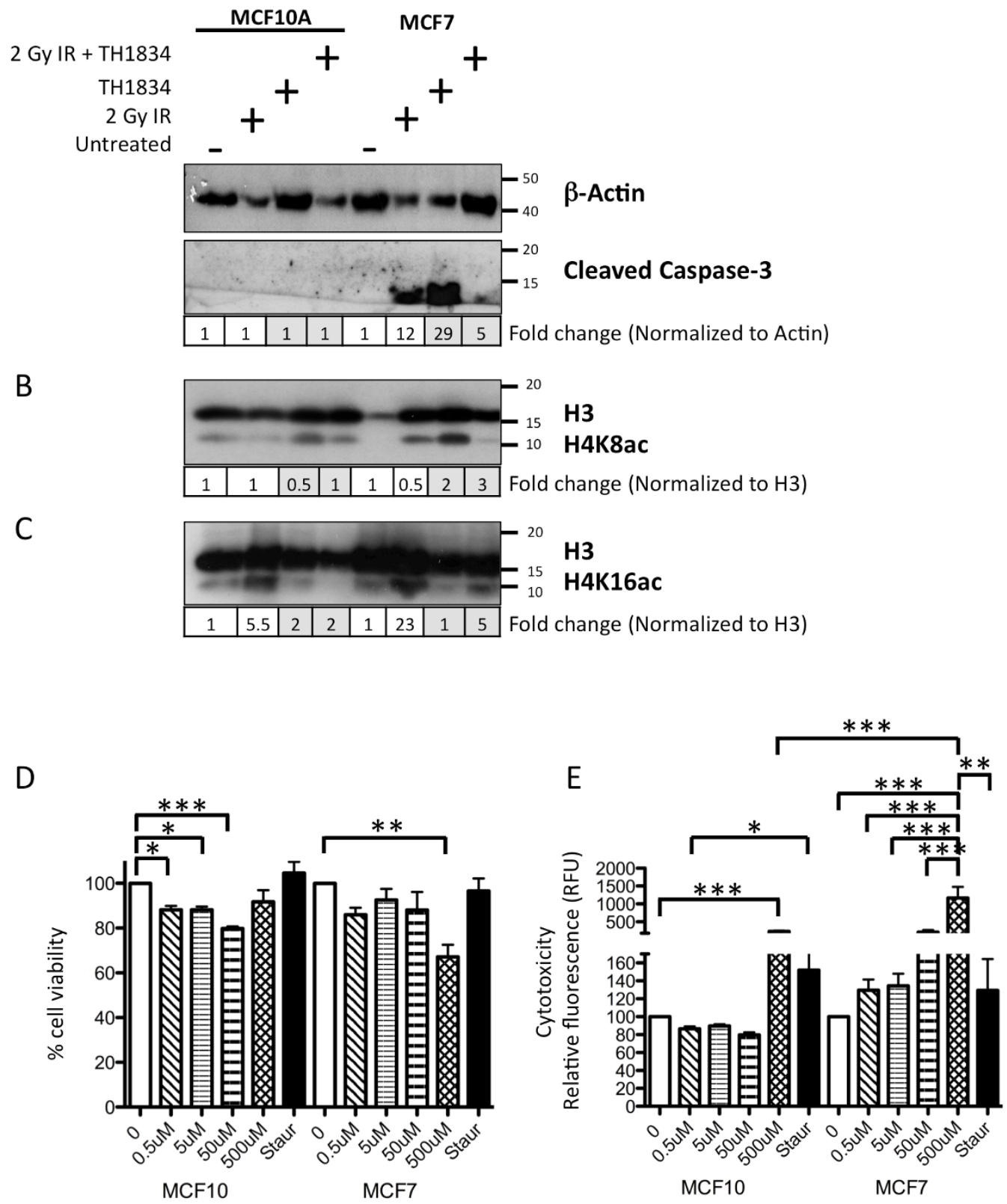

Figure 5 | TH1834 treatment induces apoptosis in breast cancer cell lines. (A). TH1834 induces caspase 3 activation. 1 hr treatment with $500 \mu \mathrm{M}$ TH1834 induces caspase 3 cleavage in breast cancer cells MCF7 but not in the control cell line MCF10A. Fold changes in activity compared to untreated, normalized to $\beta$-actin loading. (B). H4K8 acetylation is not inhibited by TH1834 pre-treatment. (C). Basal H4K16 acetylation is unaffected by pretreatment with TH1834. (B-C): Fold changes above untreated, normalized to Histone H3 loading. (A-C). Cells treated with or without IR (1 hr post IR treatment), $1 \mathrm{hr}$ pre-treatment $500 \mu \mathrm{M}$ TH1834 or a combination of IR and $500 \mu \mathrm{M}$ TH1834 pre-treatment and indicated proteins measured using immunoblotting. Cropped gels/blots are shown. Immunoblots representative of 2 independent experiments. (D). 1 hr TH1834 treatment reduces cell viability. Left side: $500 \mu \mathrm{M}$ TH1834 does not significantly reduce cell viability in MCF10A cells. Right side: $500 \mu \mathrm{M}$ TH1834 significantly reduces cell viability in MCF7 cells. (E). A $1 \mathrm{hr}$ TH1834 treatment significantly increases cytotoxicity in MCF7 cells at all concentrations. $500 \mu \mathrm{M}$ TH1834 significantly increases cytotoxicity in MCF7 compared to MCF10A cells. Left side: $500 \mu \mathrm{M}$ TH1834 treatment results in a highly significant increase in cytotoxicity in MCF10A cells. Right side: $0.5-500 \mu \mathrm{M}$ TH1834 pretreatment result in highly significant increases in cytotoxicity in MCF7 cells. TH1834 cytotoxicity is significantly higher than staurosporine in MCF7 cells. (D-E): Statistical analysis, One way Anova analysis with Tukey post test. Graphs represent $\mathrm{N}=3$. A P-value $<0.05\left(^{*}\right)$ was deemed significant, $\mathrm{P}<0.01(* *)$ very significant, and a $\mathrm{P}<0.001(* * *)$, highly significant.

supported by the increase in $\gamma \mathrm{H} 2 \mathrm{AX}$ foci observed following $50 \mu \mathrm{M}$ TH1834 treatment, with partial Tip60 inhibition stimulating genome instability, leading to the increased DNA damage observed, as previously suggested ${ }^{24}$. It has been demonstrated that DNA damage induced by IR stimulates Tip60 dependent signalling ${ }^{14,15,17-19}$ and subsequently the effects of reduced Tip60 activity in combination with this increased DNA damage become pronounced. This was supported by the apoptosis observed, as indicated by caspase 3 cleavage, following the combination of TH1834 pre-treatment and low dose IR (2 Gy) (Figure 5A). The reduction in caspase 3 activation observed in the MCF7 cells following the combination of TH1834 and low dose IR (compared to TH1834 alone) treatment is likely due to the these cells undergoing rapid apoptosis (Figure 5D-E, S9), removing them from further analysis. We also observed reduced 
levels of 53BP1 foci following TH1834 treatment, indicative of a deficiency in DNA repair upon Tip60 inhibition (Figure 4B). Together with the caspase 3 activation, decreased viability and increased cytotoxicity (Figures 5 and S9) this supports the conclusion that TH1834 mediates cell death through the apoptotic pathway in cancer cell lines. The apoptotic affects of TH1834 treatment (Figure 5A, D-E, S9) are hMOF independent, as basal hMOF dependent $\mathrm{H} 4 \mathrm{~K} 16 \mathrm{ac}^{51}$ was unaffected by TH1834 treatment (Figure 4C). However, the effect of any H4K16ac changes observed following the combination of IR and TH1834 are likely due to the rapid induction of apoptosis by the TH1834 pre-treatment. Further supporting the specificity of TH1834 there is rapid induction of $\gamma \mathrm{H} 2 \mathrm{AX}$ foci following TH1834 treatment (Figure 4, S8) and a reduction in hMOF activity has been shown to result in a significant delay in $\gamma \mathrm{H} 2 \mathrm{AX}$ IRIF formation ${ }^{52}$.

Interestingly, we found that PNT treatment did not inhibit activity in our in vitro assay (Figure 3A) and increased $\gamma \mathrm{H} 2 \mathrm{AX}$ IRIF (Figure 3B). Differences between our findings and previous work ${ }^{7}$ may be due to differences in the cell lines used (human lung fibroblasts v chicken B cells) or PNT pre-treatment time (30 v $60 \mathrm{~min})$. The reported reduction in acetylation by PNT was observed in Tip60 targets, through changes to substrate acetylation from whole cell extract, while we examined the effect of PNT directly, using an in vitro assay (Figure 3A). Investigating the effects of TH1 834 treatment on cells we found an increase in basal $\gamma \mathrm{H} 2 \mathrm{AX}$ foci formation (Figure 3E-F, left side) and a reduction in 53BP1 repair foci (Figure 3D). Supporting our findings, Tip60 knockdown is reported to induce $\gamma \mathrm{H} 2 \mathrm{AX}$ foci ${ }^{18,53}$. We speculate that the increase in $\gamma \mathrm{H} 2 \mathrm{AX}$ is due to inhibition of the chromatin remodeling functions of Tip60 required for normal cellular maintenance, leading to genotoxic stress and apoptosis as previously reported ${ }^{8,54}$. TH1834 induced DNA damage was further increased when combined with IR in cancer (Figure 3F, right side) but not control cells (Figure 3E, right side). The differential effects observed are likely due to the dysregulation of appropriate cell cycle and DNA damage responses specific to cancer cells.

Furthermore, we demonstrate the inhibitory activity of TH1834 is specific to Tip60 as basal acetylation of H4K16 and H4K8 were not reduced following $\mathrm{TH} 1834$ treatment in breast cancer cells. Supporting this $\gamma \mathrm{H} 2 \mathrm{AX}$ foci were increased following TH1834 treatment which is in keeping with previously published observations in Tip60 knockdown models ${ }^{52,55}$. Furthermore, non-specific HAT targeting by TH1834 would lead to inhibition of $\gamma \mathrm{H} 2 \mathrm{AX}$ foci following $\mathrm{IR}^{52}$. In addition, inhibition of the Tip60 complex leads to persistence of $\gamma \mathrm{H} 2 \mathrm{AX}$ focis ${ }^{53}$, consistent with our observations (Figure 4C-D).

Chicken Tip60 sequence was discovered and due to the high homology with human Tip60 (97\% across the catalytic HAT domain), used in the initial proof of concept experiments (Figure 3A-B), as a non-cancer cell line for purification of large amounts of Tip60. DT40 provides a commonly used hyper-recombinational system for further analysis of Tip60 function. Obtaining the DT40 Tip60 sequence allows additional studies targeting the Tip60 alleles. Investigating this induced haploinsufficiency, mimicking the early steps proposed in cancer progression, will allow us to better understand the protective genomic role of Tip60. A haploinsufficiency model also provides a more sensitive setting to further evaluate the effects of TH1834.

Our results indicate that in silico structure-based design of inhibitors is a useful tool for producing Tip60 inhibitors. We validated this approach by designing and synthesizing a Tip60 inhibitor (TH1834). We further demonstrated that by manipulating Tip60 activity, through inhibition using TH1834, we increased the effect of ionizing radiation against cancer cell lines (MCF7, PC-3 and DU145), resulting in apoptosis. Furthermore, our data suggests the existence of a Tip60 dependent pathway necessary for cancer cell line survival. Our results demonstrate the potential therapeutic applica- tions of Tip60 inhibitors, with future work focused on using our model to produce compounds with increased potency.

1. Kaidi, A. \& Jackson, S. P. KAT5 tyrosine phosphorylation couples chromatin sensing to ATM signalling. Nature (2013) doi:10.1038/nature12201.

2. Roth, S. Y., Denu, J. M. \& Allis, C. D. Histone Acetyltransferases. Annu. Rev. Biochem. 70, 81 (2001).

3. Yang, X.-J. The diverse superfamily of lysine acetyltransferases and their roles in leukemia and other diseases. Nucleic Acids Res. 32, 959-976 (2004).

4. Johnstone, R. W. Histone-deacetylase inhibitors: novel drugs for the treatment of cancer. Nat Rev Drug Discov 1, 287-299 (2002).

5. Garber, K. HDAC inhibitors overcome first hurdle. Nat. Biotechnol. 25, 17-19 (2007).

6. Tan, J., Cang, S., Ma, Y., Petrillo, R. L. \& Liu, D. Novel histone deacetylase inhibitors in clinical trials as anti-cancer agents. J Hematol Oncol. 3, 5 (2010).

7. Kobayashi, J., Kato, A., Ota, Y., Ohba, R. \& Komatsu, K. Bisbenzamidine derivative, pentamidine represses DNA damage response through inhibition of histone H2A acetylation. Mol Cancer 9, 34 (2010).

8. Coffey, K. et al. Characterisation of a Tip60 Specific Inhibitor, NU9056, in Prostate Cancer. PLoS ONE 7, e45539 (2012).

9. Ghizzoni, M. et al. 6-alkylsalicylates are selective Tip60 inhibitors and target the acetyl-CoA binding site. Eur J Med Chem. 47, 337-344 (2012).

10. Sagar, V., Zheng, W., Thompson, P. R. \& Cole, P. A. Bisubstrate analogue structure-activity relationships for p300 histone acetyltransferase inhibitors. Bioorg Med Chem 12, 3383-3390 (2004).

11. Wu, J., Xie, N., Wu, Z., Zhang, Y. \& Zheng, Y. G. Bisubstrate Inhibitors of the MYST HATs Esal and Tip60. Bioorg Med Chem 17, 1381-1386 (2009).

12. Sapountzi, V., Logan, I. R. \& Robson, C. N. Cellular functions of TIP60. Int J Biochem Cell Biol 38, 1496-1509 (2006).

13. Ikura, T. et al. Involvement of the TIP60 histone acetylase complex in DNA repair and apoptosis. Cell 102, 463-473 (2000).

14. Sun, Y., Jiang, X., Chen, S., Fernandes, N. \& Price, B. D. A role for the Tip60 histone acetyltransferase in the acetylation and activation of ATM. Proc Natl Acad Sci USA 102, 13182-13187 (2005).

15. Sun, Y. et al. Histone $\mathrm{H} 3$ methylation links DNA damage detection to activation of the tumour suppressor Tip60. Nat Cell Biol. 11, 1376-1382 (2009).

16. Hu, Y. et al. Homozygous disruption of the Tip60 gene causes early embryonic lethality. Dev Dyn 238, 2912-2921 (2009).

17. Bird, A. W. et al. Acetylation of histone $\mathrm{H} 4$ by Esa1 is required for DNA doublestrand break repair. Nature 419, 411-415 (2002).

18. Chailleux, C. et al. Physical interaction between the histone acetyl transferase Tip60 and the DNA double-strand breaks sensor MRN complex. Biochem J 426, 365-371 (2010).

19. Murr, R. et al. Histone acetylation by Trrap-Tip60 modulates loading of repair proteins and repair of DNA double-strand breaks. Nat Cell Biol. 8, 91-99 (2006)

20. Ramaswamy, S. et al. Multiclass cancer diagnosis using tumor gene expression signatures. Proc Natl Acad Sci USA 98, 15149-15154 (2001).

21. Mattera, L. et al. The p400/Tip60 ratio is critical for colorectal cancer cell proliferation through DNA damage response pathways. Oncogene 28, 1506-1517 (2009).

22. Ramaswamy, S., Ross, K. N., Lander, E. S. \& Golub, T. R. A molecular signature of metastasis in primary solid tumors. Nat Genet 33, 49-54 (2003).

23. Halkidou, K. et al. Expression of Tip60, an androgen receptor coactivator, and its role in prostate cancer development. Oncogene 22, 2466-2477 (2003).

24. Gorrini, C. et al. Tip60 is a haplo-insufficient tumour suppressor required for an oncogene-induced DNA damage response. Nature 448, 1063-1067 (2007).

25. Kim, J. H. et al. Transcriptional regulation of a metastasis suppressor gene by Tip60 and beta-catenin complexes. Nature 434, 921-926 (2005).

26. Shi, J. et al. Disrupting the Interaction of BRD4 with Diacetylated Twist Suppresses Tumorigenesis in Basal-like Breast Cancer. Cancer Cell (2014).

27. Peters, K. M. et al. Androgen receptor expression predicts breast cancer survival: the role of genetic and epigenetic events. BMC Cancer 12, 132 (2012).

28. Subik, K. et al. The Expression Patterns of ER, PR, HER2, CK5/6, EGFR, Ki-67 and AR by Immunohistochemical Analysis in Breast Cancer Cell Lines. Breast Cancer (Auckl) 4, 35-41 (2010).

29. Shiota, M. et al. Tip60 promotes prostate cancer cell proliferation by translocation of androgen receptor into the nucleus. Prostate 70, 540-554 (2010).

30. Bhoumik, A., Singha, N., O'Connell, M. J. \& Ronai, Z. A. Regulation of TIP60 by ATF2 modulates ATM activation. J Biol Chem 283, 17605-17614 (2008).

31. Wang, J. \& Chen, J. SIRT1 regulates autoacetylation and histone acetyltransferase activity of TIP60. J Biol Chem 285, 11458-11464 (2010).

32. Legube, G. et al. Tip60 is targeted to proteasome-mediated degradation by Mdm2 and accumulates after UV irradiation. EMBO J. 21, 1704-1712 (2002).

33. Yamagata, K. \& Kitabayashi, I. Sirt1 physically interacts with Tip60 and negatively regulates Tip60-mediated acetylation of H2AX. BIOCHEM BIOPHYS RES COMMUN. 390, 1355-1360 (2009).

34. Gozdecka, M. \& Breitwieser, W. The roles of ATF2 (activating transcription factor 2) in tumorigenesis. Biochem Soc Trans 40, 230-234 (2012).

35. Bonkhoff, H. Factors Implicated in Radiation Therapy Failure and Radiosensitization of Prostate Cancer. Prostate Cancer 2012, (2012). 
36. Hlubek, F. et al. Tip60 is a cell-type-specific transcriptional regulator. J Biochem. 129, 635-641 (2001).

37. Sun, Y., Jiang, X., Chen, S. \& Price, B. D. Inhibition of histone acetyltransferase activity by anacardic acid sensitizes tumor cells to ionizing radiation. FEBS Lett 580, 4353-4356 (2006).

38. Wu, J. et al. Small molecule inhibitors of histone acetyltransferase Tip60. Bioorg. Chem. 39, 53-58 (2011).

39. Krieger, E., Darden, T., Nabuurs, S. B., Finkelstein, A. \& Vriend, G. Making optimal use of empirical energy functions: force-field parameterization in crystal space. Proteins 57, 678-683 (2004).

40. Wang, J., Cieplak, P. \& Kollman, P. A. How well does a restrained electrostatic potential (RESP) model perform in calculating conformational energies of organic and biological molecules? J Comput Chem 21, 1049-1074 (2000).

41. Duan, Y. et al. A point-charge force field for molecular mechanics simulations of proteins based on condensed-phase quantum mechanical calculations. J Comput Chem 24, 1999-2012 (2003).

42. Jakalian, A., Jack, D. B. \& Bayly, C. I. Fast, efficient generation of high-quality atomic charges. AM1-BCC model: II. Parameterization and validation. J Comput Chem 23, 1623-1641 (2002).

43. Darden, T., York, D. \& Pedersen, L. Particle mesh Ewald: An N• $\log (\mathrm{N})$ method for Ewald sums in large systems. J Chem. Phys. 98, 10089 (1993).

44. Labute, P. The generalized Born/volume integral implicit solvent model: estimation of the free energy of hydration using London dispersion instead of atomic surface area. J Comput Chem 29, 1693-1698 (2008).

45. Brown, J. A., Eykelenboom, J. K. \& Lowndes, N. F. Co-mutation of histone H2AX S139A with Y142A rescues Y142A-induced ionising radiation sensitivity. FEBS Open Bio 2, 313-317 (2012).

46. Marck, C. 'DNA Strider': a "C"program for the fast analysis of DNA and protein sequences on the Apple Macintosh family of computers. Nucleic Acids Res. 16, 1829-1836 (1988)

47. Brown, J. A. L., Bourke, E., Liptrot, C., Dockery, P. \& Morrison, C. G. MCPH1/ BRIT1 limits ionizing radiation-induced centrosome amplification. Oncogene (2010) doi:10.1038/onc.2010.302.

48. Kamentsky, L. et al. Improved structure, function and compatibility for CellProfiler: modular high-throughput image analysis software. Bioinformatics 27, 1179-1180 (2011)

49. Marchler-Bauer, A. et al. CDD: a Conserved Domain Database for the functional annotation of proteins. Nucleic Acids Res. 39, D225-9 (2011).

50. Yan, Y., Harper, S., Speicher, D. W. \& Marmorstein, R. The catalytic mechanism of the ESA1 histone acetyltransferase involves a self-acetylated intermediate. Nat Struct Biol 9, 862-869 (2002).

51. Taipale, M. et al. hMOF histone acetyltransferase is required for histone $\mathrm{H} 4$ lysine 16 acetylation in mammalian cells. Mol Cell Biol. 25, 6798-6810 (2005).

52. Sharma, G. G. et al. MOF and Histone H4 Acetylation at Lysine 16 Are Critical for DNA Damage Response and Double-Strand Break Repair. Mol Cell Biol. 30, 3582-3595 (2010).
53. Jha, S., Shibata, E. \& Dutta, A. Human Rvb1/Tip49 is required for the histone acetyltransferase activity of Tip60/NuA4 and for the downregulation of phosphorylation on H2AX after DNA damage. Mol Cell Biol. 28, 2690-2700 (2008).

54. Ikura, T. et al. DNA Damage-Dependent Acetylation and Ubiquitination of H2AX Enhances Chromatin Dynamics. Mol Cell Biol. 27, 7028-7040 (2007).

55. Gupta, A. et al. Involvement of human MOF in ATM function. Mol Cell Biol. 25, 5292-5305 (2005).

\section{Acknowledgments}

N.F.L. and J.A.L.B. funded by Science Foundation Ireland (Grant No. SFI 07/IN.1/B958) M.S. and T.H. were funded by The Swedish Research Council, European Research Council, The Swedish Cancer Society and the Torsten and Ragnar Söderberg Foundations. E.B. funded by the National University of Ireland Galway Millennium Fund. The authors wish to thank Dr Stephan Rea for providing the histone acetylation antibodies and Dr. Carin Larsson (Stockholm University, Organisk Kemi) for provision of high-resolution mass-spectroscopy (HRMS) and Dr S. Glynn and Dr Róisín Dwyer for critical comments.

\section{Author contributions}

C.G.: Computational Experimental design, collection and assembly of data, Data analysis and interpretation, Manuscript writing. E.B.: Collection and/or assembly of data, Data analysis and interpretation, manuscript writing. M.S.: Compound synthesis, Collection and/or assembly of data, Data analysis and interpretation, manuscript writing. M.A.F. and T.K.: Collection and/or assembly of data. T.H.: Manuscript writing. L.A.E.: Data analysis and interpretation, Manuscript writing and final approval. N.L.: Conception and design, manuscript writing and final approval and J.A.L.B.: Conception and design, Collection and assembly of data, Data analysis and interpretation, Manuscript writing and final approval.

\section{Additional information}

Supplementary information accompanies this paper at http://www.nature.com/ scientificreports

Competing financial interests: The authors declare no competing financial interests.

How to cite this article: Gao, C.X. et al. Rational design and validation of a Tip60 histone acetyltransferase inhibitor. Sci. Rep. 4, 5372; DOI:10.1038/srep05372 (2014).

This work is licensed under a Creative Commons Attribution-NonCommercialNoDerivs 4.0 International License. The images or other third party material in this article are included in the article's Creative Commons license, unless indicated otherwise in the credit line; if the material is not included under the Creative Commons license, users will need to obtain permission from the license holder in order to reproduce the material. To view a copy of this license, visit http:// creativecommons.org/licenses/by-nc-nd/4.0/ 